\title{
Symbolic Model Based Testing for Component Oriented Systems
}

\author{
Alain Faivre ${ }^{1}$, Christophe Gaston ${ }^{1}$, and Pascale Le Gall ${ }^{2, \star}$ \\ ${ }^{1}$ CEA LIST Saclay F-91191 Gif sur Yvette \\ \{alain.faivre, christophe.gaston\}@cea.fr \\ ${ }^{2}$ Université d'Évry, IBISC - FRE CNRS 2873, \\ 523 pl. des Terrasses F-91000 Évry \\ pascale.legall@ibisc.univ-evry.fr
}

\begin{abstract}
In a component oriented approach, components are designed, developed and validated in order to be widely used. However one cannot always foresee which specific uses will be made of components depending on the system they will constitute. In this paper we propose an approach to test each component of a system by extracting accurate behaviours using information given by the system specification. System specifications are defined as input/output symbolic transition systems structured by a communication operator (synchronized product) and an encapsulation operator (hiding communication channels). By projecting symbolic execution of a system on its components, we derive unitary symbolic behaviours to be used as test purposes at the component level. In practice, those behaviours can be seen as typical behaviours of the component in the context of the system. We will illustrate on an example that those behaviours could not have been extracted by reasoning uniquely at the component level.
\end{abstract}

Keywords: component based system, ioco-based conformance testing, input/output symbolic transition system, symbolic execution.

\section{Introduction}

In the framework of reactive systems, a component oriented system is constituted of components continuously interacting together and with their environment by means of communication mechanisms. In a first step, basic components are usually specified, implemented and tested: this is called unitary testing. Then, the complete system is specified, implemented and tested taking into account the component based structure: this is called integration testing. Concerning integration testing, two main approaches can be followed depending on the targeted fault model. In the first approach, the global system is tested according to behaviors involving communication mechanisms, focusing on cases for which those mechanisms are not observable (i.e internal communications). Obviously, this approach is used when the targeted fault model

\footnotetext{
* This work was partially supported by the RNRT French project STACS and the RNTL French project EDEN2 .
} 
mainly deals with communication mechanisms as in 951]. In the second approach, the global system is tested by selecting behaviors of basic components that are typically activated in the system. It amounts to re-enforce unitary testing with respect to those behaviors. In terms of fault model, the counterpart of this approach is that communication mechanisms are supposed to be correctly implemented and correctly used by programmers. Thus, in this case, a non conformance of the system should only result of uncorrect implementations of components. [13] has proposed a theoretical framework based on these assumptions and has stated results concerning preservation of conformance through component composition. In this contribution, our objective is to re-enforce testing of components and intermediate sub-systems. Now, the question is: how to choose behaviors to re-enforce component and sub-system testing in order to make them more reliable in the context of the system? In fact, when a sub-system is involved in a more complex one, it is very probable that all the sub-system behaviors are not activated. In this paper, the models that we use to denote specifications of communicating systems are made of simple input/output symbolic transition systems (IOSTS) (463]) for denoting basic components, and of two structuring operators, namely composition and hiding (as in [13]). Those models based on input/output symbolic transition systems are equipped with naming mechanisms that allow us to easily retrieve all relevant information concerning sub-systems. Those naming mechanisms together with symbolic execution technics 7 are used to define relevant behaviors of sub-systems. Moreover, we show how to use those behaviors as test purposes in an ioco-based 111234 conformance testing framework. From a technical point of view, this contribution is an extension of the one presented in 4 for component oriented system testing. As we do not make any assumption concerning the communication mechanisms, a system (implementation) is considered as conformant with respect to a structured specification if it has the same structure, if for each intermediate subspecification, there exists a subsystem corresponding to it, and if each subsystem is conformant according to the ioco conformance relation with respect to the corresponding subspecification.

The paper is organized as follows. In Section [2, we present the IOSTS formalism, the notion of basic component based system and the notion of (sub-)system. In Section 3, we show how to define test purposes from symbolic execution of such systems and how to project them on any sub-system. In Section 4 , we define our symbolic test purposes. Section 5 is a conclusion.

\section{Structured Input/Output Symbolic Transition Systems}

IOSTS are used to represent behaviors of reactive systems. Those behaviors are composed of internal actions and communication actions which are emissions or receptions of values through channels. Internal states are modeled by assignments of particular variables called attributes. 


\subsection{Basic Definitions of IOSTS}

We use the following set theory notations. The set of functions of domain $A$ and codomain $B$ is denoted $B^{A}$. Џ stands for the disjoint union.

For any set $X$, Ident $_{X}$ denotes the identity function on $X$. For any two functions $f: A \rightarrow B$ and $g: C \rightarrow D$ such that $A \cap C=\emptyset, f \mid g: A \cup C \rightarrow B \cup D$ is the function such that $f \mid g(x)=f(x)$ if $x \in A$ and $f \mid g(x)=g(x)$ otherwise. Moreover, for any $E \subseteq A,\left.f\right|_{E}$ is the restriction of $f$ to $E$. A data type signature is a couple $\Omega=(S, O p)$ where $S$ is a set of type names, $O p$ is a set of operation names, each of them being provided with a profile $s_{1} \cdots s_{n-1} \rightarrow s_{n}$ (for $i \leq n$, $\left.s_{i} \in S\right)$. Let $V=\bigcup_{s \in S} V_{s}$ be a set of typed variable names. The set of $\Omega$-terms with variables in $V$ is denoted $T_{\Omega}(V)=\bigcup_{s \in S} T_{\Omega}(V)_{s}$ and is inductively defined as usual over $O p$ and $V$. Type $: T_{\Omega}(V) \rightarrow S$ is the function such that for each $t \in T_{\Omega}(V)_{s}$, Type $(t)=s$. In the following, we overload the notation Type by defining Type $(X)=s$ for any set $X \subseteq V_{s} . T_{\Omega}(\emptyset)$ is simply denoted $T_{\Omega}$. An $\Omega$-substitution is a function of $T_{\Omega}(V)^{V}$ preserving types. Any substitution may be canonically extended to terms. The set $\operatorname{Sen}_{\Omega}(V)$ of all typed equational $\Omega$ formulae contains the truth values true, false and all formulae built using the equality predicates $t=t^{\prime}$ for $t, t^{\prime} \in T_{\Omega}(V)_{s}$, and the usual connectives $\neg, \vee, \wedge$. A $\Omega$-model is a family $M=\left\{M_{s}\right\}_{s \in S}$ with, for each $f: s_{1} \cdots s_{n} \rightarrow s \in O p$, a function $f_{M}: M_{s_{1}} \times \cdots \times M_{s_{n}} \rightarrow M_{s}$. We define $\Omega$-interpretations over $V$ as applications of $M^{V}$ preserving types, that are also extended to terms of $T_{\Omega}(V)$. A model $M$ satisfies a formula $\varphi$, denoted by $M \models \varphi$, if and only if, for all interpretations $\nu, M \models_{\nu} \varphi$, where $M={ }_{\nu} t=t^{\prime}$ iff $\nu(t)=\nu\left(t^{\prime}\right)$, and where the truth values and the connectives are handled as usual. Given a model $M$ and a formula $\varphi, \varphi$ is said satisfiable in $M$, if there exists an interpretation $\nu$ such that $M \models{ }_{\nu} \varphi$. In the sequel, we suppose that data types of our IOSTS correspond to the generic signature $\Omega=(S, O p)$ and are interpreted in a fixed model $M$.

IOSTS-signatures are composed of a set of particular variables called Attributes and of a set of Channel names.

Definition 1. (IOSTS-signature) An IOSTS-signature is a couple (Att, Chan $)$ such that $A t t=\bigcup_{s \in S} A t t_{s}$. For any two IOSTS-signatures $\Sigma_{i}=\left(A t t_{i}\right.$, Chan $\left.{ }_{i}\right)$ with $i \in\{1,2\}$, the union of $\Sigma_{1}$ and $\Sigma_{2}$, denoted $\Sigma_{1} \cup \Sigma_{2}$ is the IOSTSsignature $\left(\right.$ Att $_{1} \amalg A t t_{2}$, Chan $_{1} \cup$ Chan $\left._{2}\right)$.

Union of signatures does not collapse attributes. Even though $A t t_{1}$ and $A t t_{2}$ contain a common variable name $x$, the union $\Sigma_{1} \cup \Sigma_{2}$ distinguishes the two occurrences of $x$. On the contrary, channel names are used to synchronize communication actions and thus, are shared by a simple identification in the union.

Definition 2. (Actions) The set of communication actions over $\Sigma=(A t t$, Chan), denoted $\operatorname{Act}(\Sigma)$, is the set Input $(\Sigma) \cup \operatorname{Output}(\Sigma) \cup\{\tau\}$, where:

Input $(\Sigma)=\left\{c ? Y \mid c \in\right.$ Chan, $\exists s \in S, Y \subset$ Att $\left._{s}\right\}$

Output $(\Sigma)=\left\{c ! t \mid c \in\right.$ Chan, $t \in T_{\Omega}($ Att $\left.)\right\}$

$c ? Y$ denotes the awaiting of a value to be received through the channel $c$ and to be stored on all variables of $Y$. In the sequel, when $Y$ is a singleton $\{y\}$, we 
can note $c ? y$ instead of $c ?\{y\}$. $c ! t$ denotes the emission of the value $t$ through the channel $c$ and $\tau$ is an internal action without any communication action.

We enrich basic-IOSTS of [4 with a naming mechanism associating to each transition a name chosen in a set $T N$ of transition names.

Definition 3. (IOSTS ) An IOSTS over a signature $\Sigma=($ Att, Chan) is a triple $G=($ State, init,Trans) defined by a set State of state names, an initial state init $\in$ State, and a set of transitions Trans $\subseteq T N \times($ State $\times \operatorname{Act}(\Sigma) \times$ $\operatorname{Sen}_{\Omega}(A t t) \times T_{\Omega}(A t t)^{A t t} \times$ State $) . \mathcal{S T} \mathcal{S}$ denotes the set of all IOSTS.

In the sequel, for any transition $\operatorname{tr}$ of the form $\left(n,\left(q, a c t, \varphi, \rho, q^{\prime}\right)\right)$, name $(t r)$ stands for $n$ and is called the name of $t r$, source $(t r)$ (resp. target $(t r)$ ) stands for $q$ (resp. $q^{\prime}$ ) and is called the source state of $t r$ (resp. target state of $\left.t r\right)$, act $(t r)$ stands for act and is called the communication action of tr, guard(tr) stands for $\varphi$ and is called the guard of $t r$, subst $(t r)$ stands for $\rho$ and defines how the attributes are modified when the transition is fired. Finally, body $(t r)$ stands for $\left(q, \operatorname{act}, \varphi, \rho, q^{\prime}\right)$. For an IOSTS $G, \operatorname{Sig}(G), \operatorname{Att}(G), \operatorname{Chan}(G), \operatorname{State}(G), \operatorname{init}(G)$ and Trans $(G)$ resp. stand for $\Sigma$, Att, Chan, State, init and Trans.

Definition 4. (Runs of a transition) With notations of Def. 3, let $t r \in$ Trans. Let us note Act $(M)=($ Chan $\times\{?, !\} \times M) \cup\{\tau\}$. The set Run $(t r) \subseteq$ $M^{A t t} \times A c t(M) \times M^{A t t}$ of execution runs oftr is s. $t .\left(\nu^{i}, \operatorname{act}_{M}, \nu^{f}\right) \in R u n(t r)$ iff:

- if act (tr) is of the form c!t (resp. $\tau$ ) then $M \models \nu_{\nu^{i}} \operatorname{guard}(t r), \nu^{f}=\nu^{i} \circ$ subst $(t r)$ and $a c t_{M}=c ! \nu^{i}(t) \quad\left(\right.$ resp. act $\left._{M}=\tau\right)$,

- if act(tr) is of the form $c$ ?Y then $M \models{ }_{\nu^{i}}$ guard(tr), there exists $\nu^{a}$ such that $\nu^{a}(z)=\nu^{i}(z)$ for all $z \notin Y$ and for any $x, y \in Y \nu^{a}(x)=\nu^{a}(y)$, $\nu^{f}=\nu^{a} \circ \operatorname{subst}(t r)$ and $a c t_{M}=c ? \nu^{a}(y)$ for an arbitrary $y \in Y$.

For $r=\left(\nu^{i}, \operatorname{act}_{M}, \nu^{f}\right)$, we note source $(r)$, act $(r)$, target $(r) \operatorname{resp} . \nu^{i}, \operatorname{act}_{M}, \nu^{f}$.

As in $[3$, we will use $\delta$ ! to denote under which semantic conditions an IOSTS is quiescent: quiescence refers to situations for which it is not possible to fire an output transition but only possibly input transitions or $\tau$ transitions.

Definition 5. (Suspension traces and IOSTS semantics) The set of finite paths in $G$, denoted $F P(G)$ contains all finite sequence $p=t r_{1} \ldots t r_{n}$ of transitions in $\operatorname{Trans}(G)$ such that source $\left(\operatorname{tr}_{1}\right)=\operatorname{init}(G)$ and for all $i<n$, $\operatorname{target}\left(\operatorname{tr}_{i}\right)=\operatorname{source}\left(\operatorname{tr}_{i+1}\right)$. The set of runs of $p$ denoted Run $(p)$ is the set of sequences $r=r_{1} \ldots r_{n}$ such that for all $i \leq n, r_{i}$ is a run of $t r_{i}$ and for all $i<n$, target $\left(r_{i}\right)=\operatorname{source}\left(r_{i+1}\right)$. We note $\operatorname{Tr}(r)=\operatorname{act}\left(r_{1}\right) \ldots$ act $\left(r_{n}\right)$. The set of suspension traces of a run $r$ of a finite path $p$, with $r \in \operatorname{Run}(p)$, denoted $\operatorname{STr}(p, r)$ is the least set $s . t$.:

- If $p$ can be decomposed as $p^{\prime} . t r$ with $\operatorname{tr} \in \operatorname{Trans}(G)$ and with $r$ of the form $r^{\prime} . r_{t r}$ with $r_{t r} \in R u n(t r)$, then $\left\{\right.$ m.act $\left.\left(r_{t r}\right) \mid m \in S \operatorname{Sr}\left(p^{\prime}, r^{\prime}\right)\right\} \subseteq S \operatorname{Sr}(p, r)$.

- If there exists no finite path p. $p^{\prime}$ for which there exists $r . r_{1} \cdots r_{k} \in$ Run $\left(p . p^{\prime}\right)$ with for all $i \leq k-1$, act $\left(r_{i}\right)=\tau$ and act $\left(r_{k}\right)=c ! m$ for some $c$ and $m$, then for any $\delta_{m} \in\{\delta !\}^{*}, \operatorname{Tr}(r) . \delta_{m} \in \operatorname{STr}(p, r)$.

${ }^{1} A^{*}$ denotes the set of finite sequences of elements of $A$. 
The set of suspension traces of a path $p$ is $S \operatorname{Tr}(p)=\bigcup_{r \in \operatorname{Run}(p)} S \operatorname{Tr}(p, r)$ and semantics of $G$ are $\operatorname{STr}(G)=\bigcup_{p \in F P(G)} \operatorname{STr}(p)$.

\subsection{Systems}

We introduce the concept of library which intuitively allows us to characterize a set of IOSTS denoting basic components from which systems can be built. Formally a library is a set of couples, each of them being constituted of an IOSTS name and an IOSTS definition. IOSTS names are chosen in a given set $B N$ whose elements are called basic-IOSTS names.

Definition 6. (Library) $A$ library is a set $\mathcal{B}$ whose elements are of the form $(n, G)$ where $n \in B N$ and $G \in \mathcal{S} \mathcal{T} \mathcal{S}$, s. t. for any two $\left(n_{1}, G_{1}\right),\left(n_{2}, G_{2}\right)$ in $\mathcal{B}$, $n_{1}=n_{2}$ iff $G_{1}=G_{2}$. If $G_{1} \neq G_{2}$, for any $t_{1} \in \operatorname{Trans}\left(G_{1}\right)$ and $t_{2} \in \operatorname{Trans}\left(G_{2}\right)$, name $\left(t_{1}\right) \neq$ name $\left(t_{2}\right)$. Elements of a library are called basic-IOSTS.

In the sequel we consider a library $\mathcal{B}$ and we note $B N(\mathcal{B})=\{n \mid(n, G) \in \mathcal{B}\}$ and $\operatorname{Chan}(\mathcal{B})=\{c \mid \exists(n, G) \in \mathcal{B}, c \in C h a n(G)\}$. A system over a library $\mathcal{B}$ is built from IOSTS of $\mathcal{B}$ using two structuring mechanisms: composition which is used to aggregate two systems by connecting common channels and hiding is used to internalize some channels inside the system (they are no more visible from the environment). As for basic-IOSTS, we denote any system by a name and an IOSTS. The name associated to a system reflects the structure of the system. The set $S N(\mathcal{B})$ of system names over $\mathcal{B}$ is defined as follows:

- for any $n \in B N(\mathcal{B}), n \in S N(\mathcal{B})$, (a basic-IOSTS is also a system),

- for any $n_{1}, n_{2} \in S N(\mathcal{B}),\left(n_{1} \otimes n_{2}\right) \in S N(\mathcal{B})$ (corresponding to the system obtained by composing two systems named resp. $n_{1}$ and $\left.n_{2}\right)$,

- for any $n \in S N(\mathcal{B})$ and $C \subseteq \operatorname{Chan}(\mathcal{B}), \operatorname{Hide}(C, n) \in S N(\mathcal{B})$ (corresponding to the system obtained by hiding channels of $C$ in the system named $n$ ).

Intuitively, for any system, transitions introduced in its associated IOSTS are defined over transitions of basic-IOSTSs composing the system, mainly by synchronization mechanisms. In order to be able to identify basic transitions involved in system transitions, the name associated to system transitions will explicit the underlying synchronization mechanism. Therefore, those names are of the form $\left(o,\left\{i_{1}, \cdots, i_{n}\right\}\right)$ where $o$ is a name of basic output-transition or a $\tau$-transition and $i_{1}, \cdots, i_{n}$ are names of basic input-transitions (with possibly $n=0)$. Roughly speaking, the name $\left(o,\left\{i_{1}, \cdots, i_{n}\right\}\right)$ generally refers to the synchronization of a basic output-transition named $o$ with basic input-transitions named $i_{1}, \cdots, i_{n}$. Let us point out some particular cases. Any transition obtained by synchronizing input-transitions named $i_{1}, \cdots, i_{n}$ with an emission of the environment is denoted $\left(\varepsilon,\left\{i_{1}, \cdots, i_{n}\right\}\right)$ where $\varepsilon$ denotes the absence of outputtransition. Any $\tau$-transition in a system has a name of the form $(n, \emptyset)$ where $n$ is the name of some underlying basic $\tau$-transition. The set of system transition names, denoted $S T N$, is then the set $(T N \cup\{\varepsilon\}) \times 2^{T N}$ where $\varepsilon \notin T N$.

We now define systems over a library by means of three constructions: renaming to convert a basic-IOSTS into a system, composition and hiding. 
Definition 7. (Systems over $\mathcal{B})$ The set $\operatorname{Sys}(\mathcal{B})$ of systems over $\mathcal{B}$ is the subset of $S N(\mathcal{B}) \times \mathcal{I O S T S}$ defined as follows:

Renaming: For any $(n, G) \in \mathcal{B}$ and $t \in \operatorname{Trans}(G)$, let us define $\operatorname{sn}(t)=$ $($ name $(t), \emptyset)$ if act $(t)=\tau$ or act $(t) \in \operatorname{Output}(\Sigma)$ and $\operatorname{sn}(t)=(\varepsilon,\{$ name $(t)\})$ otherwise. Let us define $R(\operatorname{Trans}(G))=\bigcup_{t \in \operatorname{Trans}(G)}\{(\operatorname{sn}(t), \operatorname{bod} y(t))\}$. $(n,(\operatorname{State}(G), \operatorname{Init}(G), R(\operatorname{Trans}(G)))$ is in $\operatorname{Sys}(\mathcal{B})$.

Composition: For any two systems $\left(n_{1}, G_{1}\right)$ and $\left(n_{2}, G_{2}\right)$ of $\operatorname{Sys}(\mathcal{B})$, let us note $G=\left(\operatorname{State}\left(G_{1}\right) \times \operatorname{State}\left(G_{2}\right),\left(\operatorname{init}\left(G_{1}\right)\right.\right.$, init $\left.\left(G_{2}\right)\right)$, Trans $)$ the IOSTS over $\operatorname{Sig}\left(G_{1}\right) \cup \operatorname{Sig}\left(G_{2}\right)$ where Trans is defined as follows:

- If $\left(\left(o_{1}, i_{1}\right),\left(q_{1}, c ! t, \varphi_{1}, \rho_{1}, q_{1}^{\prime}\right)\right) \in \operatorname{Trans}\left(G_{1}\right)$ and $\left(\left(\varepsilon, i_{2}\right),\left(q_{2}, c ? Y, \varphi_{2}, \rho_{2}\right.\right.$, $\left.\left.q_{2}^{\prime}\right)\right) \in \operatorname{Trans}\left(G_{2}\right)$ and such that type $(t)=$ type $(Y)$, then $t=\left(\left(o_{1}, i_{1} \cup i_{2}\right),\left(\left(q_{1}, q_{2}\right), c ! t, \varphi_{1} \wedge \varphi_{2}, \rho_{1} \mid \rho_{2}[Y \leftarrow t],\left(q_{1}^{\prime}, q_{2}^{\prime}\right)\right)\right) \in$ Trans.

- If $\left(\left(o_{1}, i_{1}\right),\left(q_{1}, c ! t, \varphi, \rho, q_{1}^{\prime}\right)\right) \in \operatorname{Trans}\left(G_{1}\right)$, for all $q_{2} \in \operatorname{State}\left(G_{2}\right)$ let us note $\operatorname{tr}_{1}, \cdots, \operatorname{tr}_{n}$ all transitions of the form $\operatorname{tr}_{i}=\left(n_{i},\left(q_{2}, c ? Y_{i}, \varphi_{i}, \rho_{i}, q_{i}^{\prime \prime}\right)\right)$ $\in \operatorname{Trans}\left(G_{2}\right)$ for which type $\left(Y_{i}\right)=$ type $(t)$. Let us note guard $=\wedge_{i \leq n} \neg \varphi_{i}$ if $n>0$ and guard $=$ true otherwise. Then $t=\left(\left(o_{1}, i_{1}\right),\left(\left(q_{1}, q_{2}\right), c ! t, \varphi \wedge\right.\right.$ guard,$\rho \mid$ Ident $\left.\left._{\text {Att }\left(G_{2}\right)},\left(q_{1}^{\prime}, q_{2}\right)\right)\right) \in$ Trans.

- For any two transitions of the form $\left(\left(\varepsilon, i_{1}\right),\left(q_{1}, c ? Y_{1}, \varphi_{1}, \rho_{1}, q_{1}^{\prime}\right)\right) \in$ $\operatorname{Trans}\left(G_{1}\right)$, and $\left(\left(\varepsilon, i_{2}\right),\left(\left(q_{2}, c ? Y_{2}, \varphi_{2}, \rho_{2}, q_{2}^{\prime}\right)\right) \in \operatorname{Trans}\left(G_{2}\right)\right.$ such that $\operatorname{type}\left(Y_{1}\right)=\operatorname{type}\left(Y_{2}\right)$, then

$t=\left(\left(\varepsilon, i_{1} \cup i_{2}\right),\left(\left(q_{1}, q_{2}\right), c ?\left(Y_{1} \cup Y_{2}\right), \varphi_{1} \wedge \varphi_{2}, \rho_{1} \mid \rho_{2},\left(q_{1}^{\prime}, q_{2}^{\prime}\right)\right)\right) \in$ Trans.

- If $\left(\left(\varepsilon, i_{1}\right),\left(q_{1}, c ? Y, \varphi, \rho, q_{1}^{\prime}\right)\right) \in \operatorname{Trans}\left(G_{1}\right)$, for all $q_{2} \in \operatorname{State}\left(G_{2}\right)$, let us note $\operatorname{tr}_{1}, \cdots, \operatorname{tr}_{n}$ all transitions of the form $\operatorname{tr}_{i}=\left(n_{i},\left(q_{2}, c ? Y_{i}, \varphi_{i}, \rho_{i}, q_{i}^{\prime \prime}\right)\right)$ $\in \operatorname{Trans}\left(G_{2}\right)$ for which type $\left(Y_{i}\right)=$ type $(Y)$. Let us note guard $=\wedge_{i \leq n} \neg \varphi_{i}$ if $n>0$ and guard $=$ true otherwise. Then

$t=\left(\left(\varepsilon, i_{1}\right),\left(\left(q_{1}, q_{2}\right), c ? Y, \varphi \wedge\right.\right.$ guard,$\rho \mid$ Ident $\left.\left._{\text {Att }\left(G_{2}\right)},\left(q_{1}^{\prime}, q_{2}\right)\right)\right) \in$ Trans.

- If $\left(\left(o_{1}, \emptyset\right),\left(q_{1}, \tau, \varphi_{1}, \rho, q_{1}^{\prime}\right)\right) \in$ Trans $_{1}$, for all $q_{2} \in \operatorname{State}\left(G_{2}\right)$, then $\left(\left(o_{1}, \emptyset\right),\left(\left(q_{1}, q_{2}\right), \tau, \varphi_{1}, \rho \mid\right.\right.$ Ident $\left.\left._{\operatorname{Att}\left(G_{2}\right)},\left(q_{1}^{\prime}, q_{2}\right)\right)\right) \in$ Trans.

- The role of $G_{1}$ and $G_{2}$ can be permuted in all rules described above. $\left(\left(n_{1} \otimes n_{2}\right), G\right)$ is in $\operatorname{Sys}(\mathcal{B})$.

Hiding: For any $(n, G) \in \operatorname{Sys}(\mathcal{B})$, for any $C \subseteq$ Chan $(G)$, let us note $G^{\prime}=$ (State $(G)$, init $(G)$, Trans $\left.^{\prime}\right)$ where Trans' is defined as follows:

- For any $\operatorname{tr} \in \operatorname{Trans}(G)$ where act $(t r)$ is either of the form $\tau$, c!t or $c$ ?X for some $c \notin C$, then $t r \in$ Trans'.

- For any $\operatorname{tr} \in \operatorname{Trans}(G)$ where act $(t r)$ is of the form c!t with $c \in C$, then (name $(t r),($ source $(t r), \tau$, guard $(t r)$, subst $(t r)$, target $(t r))) \in$ Trans $^{\prime}$.

$\left(\operatorname{Hide}(C, n), G^{\prime}\right)$ is in $\operatorname{Sys}(\mathcal{B})$.

Systems inherit all notations from the underlying IOSTS framework: for any system sys $=(n, G), \operatorname{Sig}($ sys $)$ stands for $\operatorname{Sig}(G), \operatorname{Att}(\operatorname{Sys})$ stands for $\operatorname{Att}(G) \ldots$ In the same way, semantics of sys are the set of suspension traces of $G$ : $\operatorname{STr}($ sys $)=$ $S \operatorname{Tr}(G)$. Note that for composition, emissions and receptions are not blocking: if no transition can be synchronized with an input (resp. output)-transition $t r$, then $t r$ is synchronized with the environment. A synchronization involves at most one output-transition: when several output transitions sharing the same source 
state could be considered at the same time to define a synchronization, this leads to non-determinism. The hiding operation make unobservable actions $c ! t$ when $c$ is in $C$ but this operation is non blocking (the output-transition introducing $c ! t$ is kept by replacing the communication action by $\tau$ ). The hiding operation is blocking for inputs $c$ ? $X$ for $c$ in $C$ : corresponding transitions are simply removed in $\operatorname{Hiding}(C, n)$. We now define sub-systems involved in a given system.

Definition 8. (Sub-systems) Let $(n, G) \in S y s(\mathcal{B})$. The set of sub-systems of $(n, G)$ denoted $\operatorname{SubS}((n, G)) \subseteq \operatorname{Sys}(\mathcal{B})$ is inductively defined as follows:

- If $n \in B N$ then $\operatorname{SubS}((n, G))=\{(n, G)\}$,

- If $n$ is of the form $n_{1} \otimes n_{2}$ then $\operatorname{SubS}((n, G))=\{(n, G)\} \cup \operatorname{SubS}\left(\left(n_{1}, G_{1}\right)\right) \cup$ $\operatorname{SubS}\left(\left(n_{2}, G_{2}\right)\right)$ where $\left(n_{1}, G_{1}\right)$ and $\left(n_{2}, G_{2}\right)$ belongs to $\operatorname{Sys}(\mathcal{B})$,

- If $n$ is of the form Hide $\left(C, n^{\prime}\right)$, then $\operatorname{SubS}((n, G))=\{(n, G)\} \cup \operatorname{SubS}\left(\left(n^{\prime}, G^{\prime}\right)\right)$ where $\left(n^{\prime}, G^{\prime}\right)$ belongs to $\operatorname{Sys}(\mathcal{B})$.

For any sub-system sys $s^{\prime}$ of a system sys, we can identify for any transition $t r$ of sys the underlying transition of $s y s^{\prime}$ involved in the definition of $t r$. This transition when it exists is called the projection of $t r$ on $s y s^{\prime}$.

Definition 9. (Projection of a transition) Let sys $\in S y s(\mathcal{B})$, sys' $\in S u b S$ (sys) and $t r=((o, i), b) \in$ Trans(sys). The projection of $t r$ on sys is the transition, when it is defined, $\operatorname{tr}_{\text {sys }}=\left(\left(o^{\prime}, i^{\prime}\right), b^{\prime}\right) \in \operatorname{Trans}\left(G^{\prime}\right)$ s. $t . o^{\prime}=o$ or $o^{\prime}=\varepsilon$ and $i^{\prime} \subseteq i$.

The naming mechanism for system transitions in Definition 7 makes $\left(\left(o^{\prime}, i^{\prime}\right), b^{\prime}\right)$ unique when it exists. Intuitively, the name $(o, i)$ captures all the subparts of the system whose state is modified by firing the transition $t r$. In particular, if $(o, i)$ does not include names of transitions issued from the sub-system sys ${ }^{\prime}$, it simply means that there is no modification of the state concerning the sub-system sys', and thus that there does not exist a corresponding transition $t r_{s y s^{\prime}}$.

\subsection{An Example of a Slot Machine}

We consider a simple slot machine, named $S$ and presented in Figure 11. The player can enter a bet into the slot machine and if he/she wins, he/she gets back the amount of his/her bet multiplied by 10 . The system $S$ is built from two basic-IOSTS, named resp. Int and $S M$ for Interface and SlotMachine. Those two basic-IOSTS are composed and some channels, used for internal communications, are hidden. Thus the name of $S$ is of the form $\operatorname{Hiding}(C, \operatorname{Int} \otimes S M)$ where:

- Int corresponds to the basic interface IOSTS between the environment (player) and the slot machine $S M$. When the system is reset (reception on int_start), the interface IOSTS waits for a bet from the player. The bet is refused when its amount is greater than 100. Otherwise, the IOSTS transmits to $S M$ the amount of the bet and then, waits for a result, win or not, from the $S M$. Depending of the result, Int transmits to $S M$ which gain should be given to the player. 
- $S M$ corresponds to the internal mechanism of the slot machine. It manages the different functionalities as appropriately updating the bank amount, deciding whether the player wins or not, and in the relevant cases, delivering cash to the player. For simplicity sake, the algorithm used to decide whether the player wins or not, is abstracted by a boolean non initialized variable $w$.

- $C$ corresponds to all the channels used by Int and $S M$ to communicate. That is, $C=\left\{i n t_{-} s t a r t, i n t \_b e t, i n t \_w i m\right.$, int_amount, int_cash $\}$.
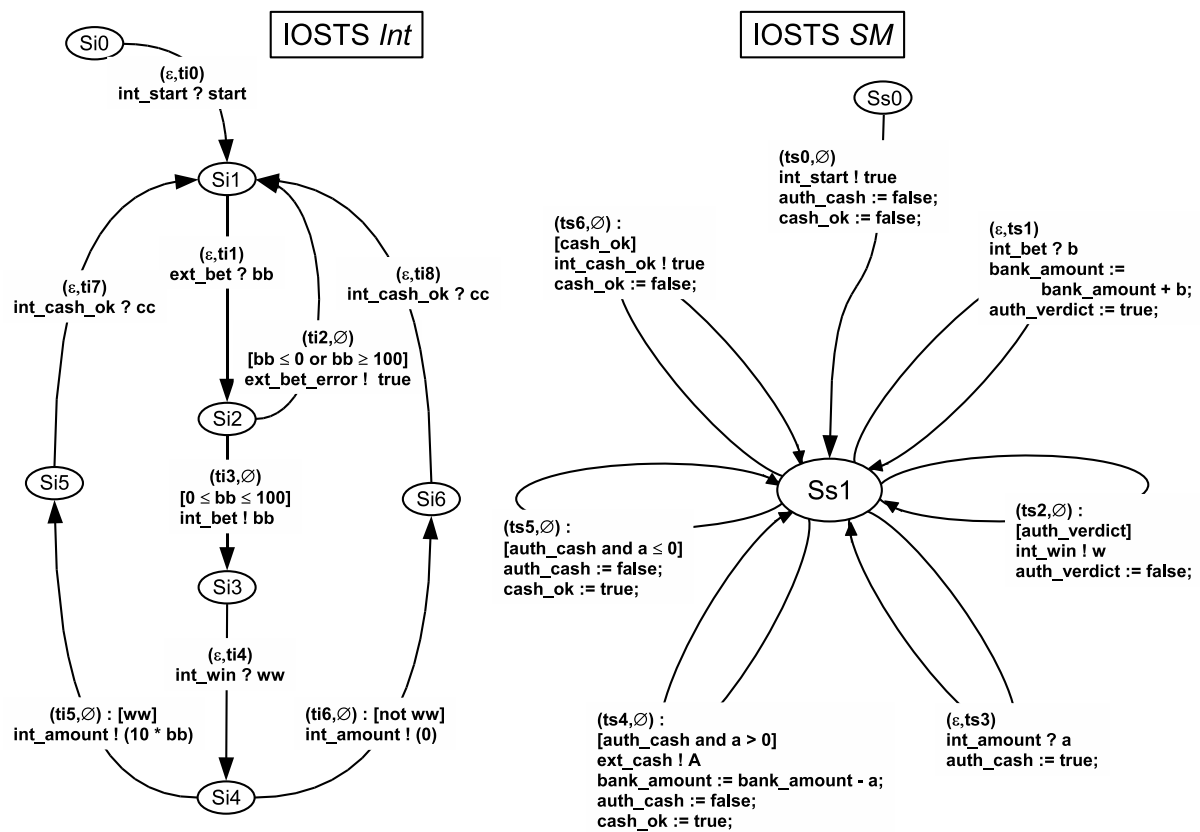

Fig. 1. An example of a slot machine

\section{$3 \quad$ System Based Test Purposes for Sub-systems}

We show how we define for any system, some test purposes dedicated to test its sub-systems. Those test purposes will capture behaviors of sub-systems that typically occur in the whole system. This is done by combining symbolic execution technics and projection mechanisms.

\subsection{Symbolic Execution}

We call a symbolic behavior of a system sys any finite path $p$ of sys for which $S \operatorname{Tr}(p) \neq \emptyset$. In order to characterize the set of suspension traces of a symbolic behavior we propose to use a symbolic execution mechanism. Symbolic execution has been first defined for programs [7] and mainly consists in replacing concrete input values and initialization values of variables by symbolic ones in order to 
compute constraints induced on these variables by the execution of the program. Symbolic execution applied to IOSTS-based systems follows the same intuition considering guards of transitions as conditions and assignments together with communication actions as instructions. Herein, symbolic execution is presented as an adaptation of 4 . In the sequel, we assume that a set of fresh variables $F=\bigcup_{s \in S} F_{s}$ disjoint from the set of attribute variables $\coprod_{(n, G) \in \mathcal{B}} \operatorname{Att}(G)$ is given. We now give the intermediate definition of symbolic extended state which is a structure allowing to store information about a symbolic behavior: the system current state (target state of the last transition of the symbolic behavior), the path condition which characterizes a constraint on symbolic variables to reach this state, and the symbolic values associated to attribute variables. As compared to 4, we also add a fourth stored information: it is given in the form of a constraint on symbolic variables which is not computed during the symbolic execution of the system. It is called an external constraint and in practice it will be inherited from a projection mechanism.

Definition 10. (Symbolic extended state) $A$ symbolic extended state of sys is a quadruple $\eta=(q, \pi, f, \sigma)$ where $q \in \operatorname{State}($ sys $), \pi \in \operatorname{Sen}_{\Omega}(F)$ is called a path condition, $f \in \operatorname{Sen}_{\Omega}(F)$ is called an external constraint and $\sigma \in T_{\Omega}(F)^{\text {Att }(\text { sys })}$ is called a symbolic assignment of variables. $\eta=(q, \pi, f, \sigma)$ is said to be satisfiable if $\pi \wedge f$ is satisfiabl 2 . One notes $\mathcal{S}($ sys $)$ (resp. $\mathcal{S}_{\text {sat }}($ sys $\left.)\right)$ the set of all the (resp. satisfiable) symbolic extended states over $F$.

For any symbolic extended state $\eta$ of the form $(q, \pi, f, \sigma), q$ is denoted state $(\eta)$, $\pi$ is denoted $p c(\eta), \sigma$ is denoted $\operatorname{sav}(\eta)$ (for symbolic assignment of variables) and $f$ is denoted $e c(\eta)$. Now, we show how to give symbolic counterparts to transitions of a system. The idea is to consider any symbolic extended state defined over the source state of the transition, and to construct a new target symbolic extended state defined over the target state of the transition. The external constraint of the target symbolic extended state is a conjunction formed with the external constraint of the source symbolic extended state and a new external constraint (denoted $c t$ in the following Definition). In the sequel, for any system sys, Sig (sys, F) stands for the signature $(F$, Chan $($ sys $))$.

Definition 11. (Symbolic execution of a transition) With notations of Definition [10, for any $\eta \in \mathcal{S}($ sys $)$, for any $\operatorname{tr} \in$ Trans(sys) such that source $(t r)=$ state $(\eta)$, a symbolic execution of $t r$ from $\eta$ is a triple $s t=\left(\eta, s a, \eta^{\prime}\right) \in \mathcal{S}($ sys $) \times$ $\operatorname{Act}(\operatorname{Sig}($ sys,$F)) \times \mathcal{S}($ sys $)$ such that there exists $c t \in \operatorname{Sen}_{\Omega}(F)$ for which:

- if act $(\mathrm{tr})=c ! t$ then $s a$ is of the form $c ! z$ for some $z \in F$ and $\eta^{\prime}=$ $(\operatorname{target}(\operatorname{tr}), p c(\eta) \wedge \operatorname{sav}(\eta)(\operatorname{guard}(\operatorname{tr})) \wedge z=\operatorname{sav}(\eta)(t), e c(\eta) \wedge \operatorname{ct}, \operatorname{sav}(\eta) \circ$ subst $(t r))$,

- if act $(t r)=c$ ?Y then sa is of the form $c$ ? $z$ for some $z \in F$ and $\eta^{\prime}=$ $\left(\operatorname{target}(\operatorname{tr}), p c(\eta) \wedge \operatorname{sav}(\eta)(\operatorname{guard}(\operatorname{tr})), e c(\eta) \wedge c t, \operatorname{sav}(\eta) \circ(y \mapsto z)_{y \in Y} \circ \operatorname{subst}(\operatorname{tr})\right)$,

- if act $(\operatorname{tr})=\tau$ then $s a=\tau$ and $\eta^{\prime}=(\operatorname{target}(\operatorname{tr}), p c(\eta) \wedge \operatorname{sav}(\eta)(\operatorname{guard}(\operatorname{tr})), e c(\eta)$ $\wedge c t, \operatorname{sav}(\eta) \circ \operatorname{subst}(t r))$.

$\overline{{ }^{2} \text { Here } \pi \wedge f}$ is satisfiable if and only if there exists $\nu \in M^{F}$ such that $M \models{ }_{\nu} \pi \wedge f$. 
The definition of st only depends on $t r, \eta, c t$ and the chosen variable $z$. Therefore, it is conveniently denoted $S E(t r, \eta, c t, z)$ (if $a c t(t r)=\tau, z$ is useless). For any $s t=\left(\eta, s a, \eta^{\prime}\right)$, source $(s t)$ stands for $\eta$, target $(s t)$ stands for $\eta^{\prime}$ and act $(s t)$ stands for $s a$.

We now define symbolic execution of systems. Intuitively, a symbolic execution of a system sys is seen as a rooted tree whose paths are composed of sequences of symbolic executions of transitions which are consecutive in sys. The root is a symbolic extended state made of the initial state init(sys), the path condition true, an arbitrary initialization $\sigma_{0}$ of variables of $\operatorname{Att}($ sys $)$ in $F$, and an external constraint reduced to true (no constraint at the beginning of the execution). Moreover, if a transition is symbolically executed with an external constraint $c t$, then it is also executed with the external constraint $\neg c t$.

Definition 12. (Symbolic execution of a system) $A$ full symbolic execution of sys over $F$ is a triple sys symb $=(\mathcal{S}($ sys $)$, init, $R)$ with init $=($ init $($ sys $)$, true, true, $\left.\sigma_{0}\right)$ where $\sigma_{0}$ is an injective substitution in $F^{A t t(s y s)}$ and $R \subseteq \mathcal{S}($ sys $) \times$ $\operatorname{Act}(\operatorname{Sig}($ sys,$F)) \times \mathcal{S}($ sys $)$ satisfies the following properties:

- for any $\eta \in \mathcal{S}($ sys $)$, for all $t r \in$ Trans $($ sys $)$ such that source $(t r)=\operatorname{state}(\eta)$, there exists exactly two constrained symbolic executions of $t r$ in $R$ respectively of the form $S E(t r, \eta, c t, z)$ and $S E(t r, \eta, \neg c t, z)$. Those two transitions are said to be complementary.

- for any $\left(\eta^{i}, c \npreceq x, \eta^{f}\right) \in R$ with $\emptyset \in\{!, ?\}, \forall a \in \operatorname{Att}($ sys $)$, then $\sigma_{0}(a) \neq x$,

- for any $\left(\eta^{i}, c \bigsqcup x, \eta^{f}\right) \in R$ and $\left(\eta^{\prime i}, d \sharp x, \eta^{\prime}\right) \in R$ with $\natural, \sharp \in\{!, ?\}$ which are not complementary, then $x \neq y$.

The symbolic execution of sys over $F$ associated to sys symb is the triple $S E($ sys $)=\left(\mathcal{S}_{\text {sat }}(\right.$ sys $)$, init, $\left.R_{\text {sat }}\right)$ where $R_{\text {sat }}$ is the restriction of $R$ to $\mathcal{S}_{\text {sat }}($ sys $) \times$ $\operatorname{Act}(\operatorname{Sig}($ sys,$F)) \times \mathcal{S}_{\text {sat }}($ sys $)$.

We use the notation $F P(S E(s y s))$ to denote the set of finite paths of $S E(s y s)$. To define a run of a finite path $p$, we proceed as follows. We choose an interpretation $\nu: F \rightarrow M$ such that $M \models{ }_{\nu} p c\left(\eta_{f}\right) \wedge e c\left(\eta_{f}\right)$ where $\eta_{f}$ is the last symbolic extended state of $p$. Then for each $\left(\eta, a c t, \eta^{\prime}\right)$ of $p$ we associate a run $\left(\nu(\operatorname{sav}(\eta)), \operatorname{act}_{M}, \nu\left(\operatorname{sav}\left(\eta^{\prime}\right)\right)\right)$ where $a c t_{M}=\tau$ if $a c t=\tau$ and $a c t_{M}=c q \nu(z)$ if $a c t$ is of the form $c \curvearrowleft z$ with $\downarrow \in\{!, ?\}$. The sequence of such formed triples constitute a run of $p$. Note that the set of all runs of all finite paths of $\operatorname{FP}(S E(s y s))$ is exactly the set of all runs of all finite paths of sys in the sense of Definition 5 and this set is independent of the external constraints chosen to execute transitions. Those external constraints are simply used to partition symbolic behaviors. A trivial partitioning can be characterized by choosing true as external constraints for executing any transition from any symbolic state. In this case the obtained symbolic execution is isomorphic to the one described in 4] which does not contain any external constraint. Besides note that any finite path $p$ of a symbolic execution of sys characterizes a set of suspension traces obviously determined by its set of runs and the finite path corresponding to $p$ in sys (See Definition 5). Therefore any symbolic execution of sys characterizes a set of suspension traces which can be easily proven to be this associated to sys in the sense of 
Definition 5 Now, since internal actions are not observable in black box testing, we propose to eliminate them as follows.

Definition 13. ( $\tau$-reduction of a constrained symbolic execution) The $\tau$-reduction of $S E($ sys $)$ is the triple $S E(\text { sys })_{\tau}=\left(\mathcal{S}_{\text {sat }}(\right.$ sys $)$, init, $\left.R_{\text {sat }}^{\tau}\right)$, where $R_{\text {sat }}^{\tau} \subseteq \mathcal{S}_{\text {sat }}($ sys $) \times \operatorname{Act}(\operatorname{Sig}($ sys,$F)) \times \mathcal{S}_{\text {sat }}($ sys $)$ is such that for any sequence $s t_{1} \cdots s t_{n}$ where for all $i \leq n s t_{i} \in R_{\text {sat }}$ :

- for all $i \leq n-1 \operatorname{act}\left(\operatorname{st}_{i}\right)=\tau$, $\operatorname{source}\left(\right.$ st $\left._{i+1}\right)=\operatorname{target}\left(\operatorname{st}_{i}\right) \operatorname{and} \operatorname{act}\left(\operatorname{st}_{n}\right) \neq \tau$,

- either source $\left(s_{1}\right)=$ init or there exists st $\in R_{\text {sat }}$ such that target $(s t)=$ $\operatorname{source}\left(\operatorname{st}_{1}\right)$ and $\operatorname{act}(s t) \neq \tau$,

then $\left(\operatorname{source}\left(\operatorname{st}_{1}\right), \operatorname{act}\left(\operatorname{st}_{n}\right), \operatorname{target}\left(s t_{n}\right)\right) \in R_{\text {sat }}^{\tau}$.

Note that $S E($ sys $)$ and $S E(\text { sys })_{\tau}$ characterize the same suspension traces. However, we need in the sequel to be able to symbolically identify situations in which quiescence is allowed. This is done by adding symbolic transitions labeled by $\delta$ ! in the $S E(s y s)_{\tau}$.

Definition 14. (Quiescence enrichment) Quiescence enrichment of $S E$ (sys) is the triple $S E(\text { sys })_{\delta}=\left(\mathcal{S}_{\text {sat }}(\right.$ sys $)$, init, $\left.R_{\delta}\right)$ where $R_{\delta}=R_{\text {sat }}^{\tau} \cup \Delta R_{\delta}$ with $\Delta R_{\delta} \subseteq \mathcal{S}_{\text {sat }}($ sys $) \times\{\delta !\} \times \mathcal{S}_{\text {sat }}($ sys $)$ is such that for any $\eta \in \mathcal{S}_{\text {sat }}($ sys $)$, if we note out $_{\eta}=\left\{t r_{1}, \cdots, t r_{n}\right\}$ the set of all transitions $t r_{i} \in R_{\text {sat }}^{\tau}$ such that $\operatorname{act}\left(\operatorname{tr}_{i}\right) \in$ output $(\operatorname{Sig}($ sys,$F))$, if we note $f \in \operatorname{Sen}_{\Omega}(F)$ the formula of the form true if out $_{\eta}$ is empty and of the form $\bigwedge_{i \leq n} \neg\left(\right.$ pc(target $\left.\left(\operatorname{tr}_{i}\right)\right) \wedge$ ec(target $\left.\left.\left(\operatorname{tr}_{i}\right)\right)\right)$ otherwise, if we note $\eta^{\prime}=(\operatorname{state}(\eta), p c(\eta) \wedge f, \operatorname{ec}(\eta), \operatorname{sav}(\eta))$ then $\left(\eta, \delta !, \eta^{\prime}\right) \in \Delta R_{\delta}$.

An example of a slot machine: symbolic execution Figure 2 shows a sub-tree of the symbolic execution of the slot machine system presented in Figure [1. as carried out by the AGATHA tool (82]).

External constraints for any two complementary transitions are resp. true and false in the corresponding full symbolic execution. They never appear in the figure. We use the so-called inclusion criteria to end this execution. This criteria allows to stop symbolic execution when it detects that an encountered symbolic extended state is included in another already computed one. Intuitively, $(q, \pi, f, \sigma)$ is included

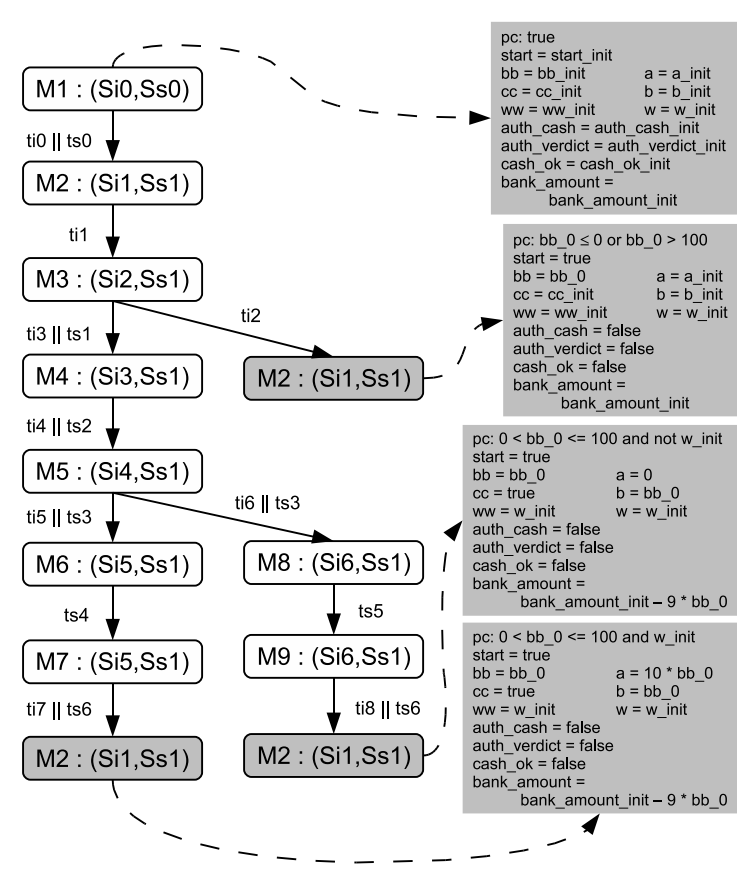

Fig. 2. Symbolic execution of the slot machine 
in $\left(q^{\prime}, \pi^{\prime}, f^{\prime}, \sigma^{\prime}\right)$ if $q^{\prime}=q$ and the constraints induced on $\operatorname{Att}($ sys $)$ by $\sigma$ and $\pi \wedge f$ are stronger than those induced by $\sigma^{\prime}$ and $\pi^{\prime} \wedge f^{\prime}$. The interested readers can refer to 104 for more formal definitions. Let us point out that the symbolic sub-tree of $S$ computes three characteristic symbolic behaviors. The left path corresponds to a winning bet, the middle path corresponds to a lost bet, and finally the right path corresponds to a forbidden bet. The initial and ending states are annotated with symbolic values of all attribute variables.

\subsection{Symbolic Behavior Projections}

For any finite path $p$ of a symbolic execution of sys and a sub-system sys $s^{\prime}$ of $s y s$, we characterize the symbolic behavior $p_{s y s^{\prime}}$ of $s y s^{\prime}$ involved in $p$. For this purpose, we begin by defining the projection of a symbolic transition.

Definition 15. (Projection of a symbolic transition) Let sys be a system of $\operatorname{Sys}(\mathcal{B})$. Let sys $s^{\prime} \in \operatorname{SubS}($ sys $)$. Let $t r \in$ Trans $($ sys $)$ such that trys $_{\text {sy }}$ is defined. Let us note $s t=S E(t r, \eta, c t, z)$ a symbolic execution of $t r$ and $\eta_{\text {sys }} \in \mathcal{S}_{\text {sat }}\left(\right.$ sys $\left.^{\prime}\right)$ such that state $\left(\eta_{s y s^{\prime}}\right)=$ source $\left(\operatorname{tr}_{\text {sys }}\right)$. The projection of st on sys of source $\eta_{\text {sys }}$ is $S E\left(\operatorname{tr}_{s y s^{\prime}}, \eta_{s y s^{\prime}}, p c(\operatorname{target}(s t)) \wedge \operatorname{ec}(\operatorname{target}(s t)), z\right)$.

The external constraint of the target state of the projection represents the constraints induced by the nature of the interactions of the sub-system with the other parts of the whole system. Now we generalize to symbolic behaviors.

Definition 16. (Projection of a path) Let $p \in F P(S E(S y s))$. The projection of $p$ on sys $s^{\prime}$ denoted $p_{\text {sys }} \in\left(\mathcal{S}_{\text {sat }}\left(S y s^{\prime}\right) \times \operatorname{Act}\left(\operatorname{Sig}\left(\operatorname{sys}^{\prime}, F\right)\right) \times \mathcal{S}_{\text {sat }}\left(\text { Sys }^{\prime}\right)\right)^{*}$ together with its associated target state denoted target $\left(p_{\text {sys }}\right)$ are inductively $m u-$ tually defined as follows:

- if $p$ is of the form st $=S E(t r$, init, ct, $z) \in R_{\text {sat }}$ then let us note $\eta_{\text {sys }}=$ $\left(\right.$ init $\left(\right.$ sys $\left.^{\prime}\right)$, true, true, sav(init) $\left.\left.\right|_{\text {Att }\left(\text { sys }^{\prime}\right)}\right)$ then $p_{\text {sys }}$ is the projection st $_{\text {sys }}$ of st on sys' of source $\eta_{\text {sys }}$ when it is defined, and in this case target $\left(p_{\text {sys }}\right)=$ target $\left(\right.$ st $\left._{\text {sys }}\right)$. Otherwise $p_{\text {sys }}$ is the empty path and target $\left(p_{\text {sys }}\right)=\eta_{\text {sys }}$.

- if $p$ is of the form $p^{\prime}$.st with st $=S E(t r, \eta, c t, z)$ then either the projection st sys $^{\prime}$ of st on sys $s^{\prime}$ of source target $\left(p_{\text {sys }}^{\prime}\right)$ is defined and: $p_{\text {sys }}=$ $p_{\text {sys }}^{\prime} . s t_{s y s^{\prime}}$ and $\operatorname{target}\left(p_{\text {sys }}\right)=\operatorname{target}\left(\right.$ st $\left._{\text {sys }}\right)$. Otherwise, $p_{\text {sys }}=p_{\text {sys }}^{\prime}$ and $\operatorname{target}\left(p_{s y s^{\prime}}\right)=\operatorname{target}\left(p_{s y s^{\prime}}^{\prime}\right)$.

Thus from any symbolic behavior of a system we can identify by projection symbolic behaviors of any sub-system whose external constraints reflect a usage of the sub-system in the whole system. Those projected behaviors are then good candidates to become behaviors to be tested on sub-systems: thus they will be chosen to construct test purposes.

\section{Symbolic Execution Based Conformance Testing}

\subsection{Conformance Testing and System-Based Test Purposes}

Model-based testing supposes that a conformance relation formally defines how are linked the specification $G$ and the system under test $S U T$. Our work is based 
on the widely used ioco relation, initially designed for labeled transition systems 11] and afterwards adapted for symbolic transition systems 6 3 4. All the iocobased testing settings consider that the $S U T$ is a black-box system which can be observed only by its behavior given as input/output sequences. These sequences of observations may include the special output $\delta$ ! indicating that the $S U T$ is in a quescient state. The set of all traces, possibly including suspension transitions, which can be observed from $S U T$ is denoted $S \operatorname{Tr}(S U T)$. When dealing with IOSTS, data handled in these sequences are concrete values denoted by ground terms of $T_{\Omega}$. By test hypothesis, the $S U T$ is modeled as a labeled transition system $S$ for which transitions are emissions (outputs), receptions (inputs) carrying concrete values and such that the set of suspension traces of $S$ coincide with $S T r(S U T)$. Moreover, as usual, the $S U T$ is supposed to accept all inputs in all states (hypothesis of input-enabled system). Intuitively a $S U T$ conforms to its specification $G$ with respect to ioco if any $S U T$ output (including $\delta$ !) is specified in $G$ provided that the sequence of input/output preceding the considered observation is also specified in $G$.

Definition 17. (ioco) An input-enabled system SUT conforms to G iff for any tra $\in S \operatorname{Tr}(G) \cap S \operatorname{Tr}(S U T)$, if there exists act $\in \operatorname{Act}(M) \cup\{\delta !\}$ of the form $c ! t$ or $\delta$ ! such that tra.act $\in S \operatorname{Tr}(S U T)$, then tra.act $\in S \operatorname{Tr}(G)$.

A test execution consists in executing a transition system, called a test case, on the SUT in order to produce test verdicts. The test case and the SUT are synchronized by coupling emissions and receptions. Test purposes are used to select some behaviors to be tested. In a previous work 4, we have proposed to model test purposes as finite trees extracted from symbolic executions of $G$. Such a symbolic execution describes all the possible behaviors of $G$. Therefore it is equivalent to test the $S U T$ by selecting paths in $G$ or in a symbolic execution of $G$. Indeed, we have demonstrated the following completeness result : if an $S U T$ does not conform to a specification $G$, then there exists a test purpose such that our corresponding testing algorithm can emit a verdict $F A I L$. The main advantage of characterizing test purposes from a symbolic execution of $G$ is that the testing process can be expressed as a simultaneous traversal of both the symbolic execution and the test purpose. Verdicts are emitted according to the fact that the observed behavior, in the form of a sequence of inputs (stimulations) and outputs (observations), does or does not belong to the test purpose and to the symbolic execution. We have defined 4 verdicts: WeakPASS when the behavior belongs to the test purpose and to at least one path of the symbolic execution which is not in the test purpose, $P A S S$ when the behavior belongs to the test purpose and not to any path of the symbolic execution which does not belong to the test purpose, INCONC (for inconclusive) when the behavior belongs to the symbolic execution and not to the test purpose, and finally $F A I L$ when the behavior belongs neither to the test purpose nor to the symbolic execution. In the sequel, we slightly adapt the framework described in 4 to our purpose. Behaviors of any sub-system sys' to be tested are obtained by projecting behaviors of a symbolic execution of the whole system. It remains to define test purposes dedicated to test such projected behaviors. As basic- 
IOSTS and hiding mechanism introduce $\tau$-transitions, then such a projected behavior $p_{\text {sys }}$ may contain $\tau$-transitions. Since such internal transitions cannot be observed during testing, we construct test purposes from a $\tau$-reduced symbolic execution enriched by quiescence. We identify all its finite paths whose last transitions are output-transitions (including $\delta$-transitions) and which result of the $\tau$-reduction of a path whose $p_{s y s^{\prime}}$ is a prefix. Those $\tau$-reduced finite paths become behaviors to be tested.

Definition 18. (Test purpose) Let $S E\left(\right.$ sys $\left.^{\prime}\right)$ be a symbolic execution of sys ${ }^{\prime}$ such that $p_{\text {sys }} \in F P\left(S E\left(s y s^{\prime}\right)\right)$. Let us note $\operatorname{ext}_{o}\left(p_{\text {sys }}\right)$ the set $\left\{p_{\text {sys }}\right\}$ if $p_{\text {sys }}$ is of the form p. $\left(\eta\right.$, act, $\left.\eta^{\prime}\right)$ with act $\in \operatorname{Output}\left(\operatorname{Sig}\left(\right.\right.$ sys $\left.\left.^{\prime}, F\right)\right)$ and whose elements are all paths of the form $p_{\text {sys }} \cdot\left(\eta_{1}\right.$, act $\left._{1}, \eta_{1}^{\prime}\right) \cdots\left(\eta_{n}\right.$, act $\left._{n}, \eta_{n}^{\prime}\right)$ with act ${ }_{i}=\tau$ for $i<n$ and act $_{n} \in \operatorname{Output}\left(\operatorname{Sig}\left(\right.\right.$ sys $\left.\left.^{\prime}, F\right)\right)$ otherwise. Let us note $T \subseteq \mathcal{S}_{\text {sat }}\left(\right.$ sys $\left.^{\prime}\right)$ the set of all the target states of all the finite paths of ext $t_{o}\left(p_{\text {sys }}\right)$. A symbolic test purpose for $p_{\text {sys }}$ and $S E\left(\right.$ sys $\left.^{\prime}\right)$ is an application $T P: \mathcal{S}_{\text {sat }} \rightarrow\{$ skip, accept, $\odot\}$ such that:

- for all $\eta \in T, T P(\eta)=$ accept,

- for all finite path $s t_{1} \cdots s t_{n}$ such that for all $i \leq n$, st $_{i} \in R_{\tau}$ and TP $\left(\operatorname{target}\left(s t_{n}\right)\right)$ = accept, then TP $\left(\right.$ source $\left.\left(s_{i}\right)\right)=$ skip,

- If $\operatorname{ext}_{o}\left(p_{\text {sys }}\right)=\left\{p_{\text {sys }}\right\}$ then all other states $\eta$ verify $T P(\eta)=\odot$,

- if $\operatorname{ext}_{o}\left(p_{\text {sys }}\right) \neq\left\{p_{\text {sys }}\right\}$ and the last transition of $p_{\text {sys }}$ is an input-transition st then if there exists a transition $s_{\delta} \in \Delta R_{\delta} s$. $t$. source $\left(s_{\delta}\right)=\operatorname{target}(s t)$ then $T P\left(\right.$ target $\left(\right.$ st $\left.\left._{\delta}\right)\right)=$ accept and all other states $\eta \in R_{\delta}$ verify $T P(\eta)=\odot$,

- if $\operatorname{ext}_{o}\left(p_{\text {sys }}\right) \neq\left\{p_{\text {sys }}\right\}$ and the last transition of $p_{\text {sys }}$ is a $\tau$-transition then all other states $\eta \in R_{\delta}$ verify $T P(\eta)=\odot$.

Definition 18 introduces the notion of symbolic test purpose, which extends the notion of test purposes as defined in 4 by considering a symbolic execution of a system which incorporates constraints issued from a surrounding system. Let us remark that constraint symbolic executions allow us to characterize test purposes in the same way: a test purpose is a finite sub-tree of a $\delta$-enriched symbolic execution whose leaves are target states of output transitions (identified by means of the labeling function which associates accept to those states). The algorithm of test case generation given in 4] can directly be applied.

An example of a slot machine: projection Let us consider $p$ the left path of Figure 2] corresponding to the winning case. In Figure 3] the left path represents $p$. The right path is the projection $p_{S M}$ of $p$ on $S M$. Nearby each symbolic extended state name $S s_{i}$ we indicate in the grey box the content of the symbolic state, up to simplifications in path conditions and external constraints for sake of readability. The behavior denoted by $p_{S M}$ corresponds intuitively to the following scenario: after the initialization, a bet is received for amount greater to 0 and less or equal to 100 (this is a constraint induced by the interface). Then $S M$ sends a verdict stating that the player has won, the value to be removed of the bank account is received and correspond to 10 times the bet. The amount is sent to the interface and effectively removed from the bank account. Finally, 
$S M$ sends an ending operation message to the Int. A test purpose $L$ for this behavior would label $N_{6}$ by accept and $N 0$ to $N 5$ by skip. On the right part of the figure, $N^{\prime} 2$ and $N^{\prime} 4$ are target states of the complementary transitions of respectively ( $N \_1$, int_bet?bb_0, $\left.N \_2\right)$ and $\left(N \_3\right.$, int_amount?a_0, N4). $N^{\prime} 2$ characterizes cases for which the received bet is out of the range allowed by the interface. $N_{4}^{\prime}$ characterizes situation for which the gain does not correspond to 10 times the bet contrarily to the information sent by the interface.

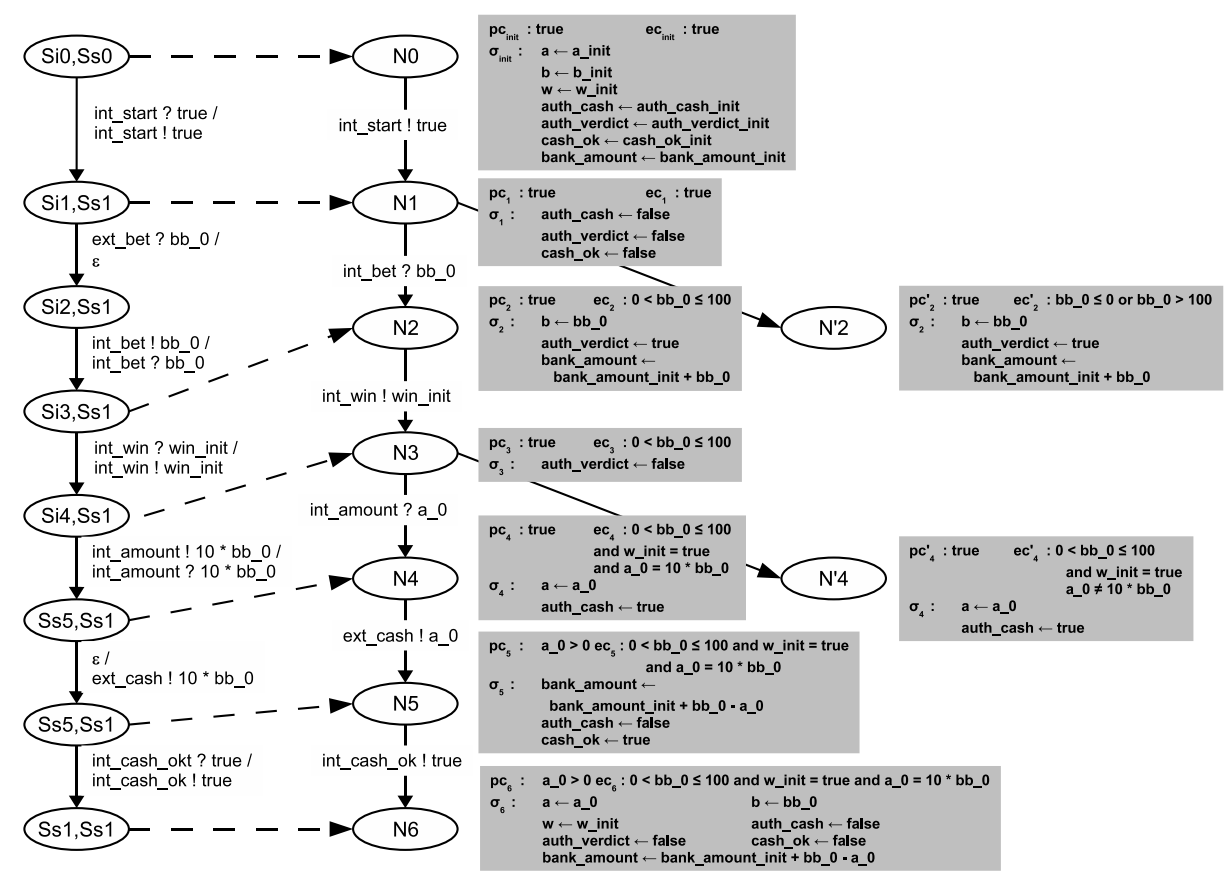

Fig. 3. Projection in the example of the slot machine

Those two situations are possible for $S M$ but note relevant in the frame of the whole system. Therefore $L$ would label $N^{\prime} 2$ and $N^{\prime} 4$ with $\odot$. To conclude, let us point out that such a test purpose cannot be deduced only from the knowledge of $S M$ : it clearly depends on the way $S M$ is used in the whole system $S$. This exemplifies our initial goal of eliciting from a system dedicated test purposes for each subsystem.

\section{Conclusion and Future Works}

We have extended the framework of IOSTS introduced in 4], in order to deal with component-based system specifications and we have used symbolic execution mechanisms in order to compute behaviors of sub-systems constrained by systems in which they are involved. Then, we have defined test purposes from 
those constrained behaviors. The definition of dedicated methodologies for component based systems should clearly rely on the targeted fault models. We plan to study fault models that mainly deal with communication mechanisms as in [5]. For such fault models, a testing methodology would probably preconize to construct test purposes for behaviors involving a lot of internal communication synchronizations. Besides, we also plan to target fault models that mainly deal with basic components. As in [13], we could consider that composition and hiding mechanisms are well implemented such that an appropriate testing methodology would only consider test purposes directly defined at the component level. More generally, our next goal is to provide testing methodologies for component based systems which take advantage of the fact that some components or subsystems have been previously intensively tested such that a large class of tests becomes useless in the context of the whole system.

\section{References}

1. Berrada, I., Castanet, R., Félix, P.: Testing Communicating Systems: a Model, a Methodology, and a Tool. In: Khendek, F., Dssouli, R. (eds.) TestCom 2005. LNCS, vol. 3502, pp. 111-128. Springer, Heidelberg (2005)

2. Bigot, C., Faivre, A., Gallois, J.-P., Lapitre, A., Lugato, D., Pierron, J.-Y., Rapin, N.: Automatic test generation with AGATHA. In: Garavel, H., Hatcliff, J. (eds.) ETAPS 2003 and TACAS 2003. LNCS, vol. 2619, pp. 591-596. Springer, Heidelberg (2003)

3. Frantzen, L., Tretmans, J., Willemse, T.A.C.: A Symbolic Framework for ModelBased Testing. In: Havelund, K., Núñez, M., Roşu, G., Wolff, B. (eds.) Formal Approaches to Software Testing and Runtime Verification. LNCS, vol. 4262, pp. 40-54. Springer, Heidelberg (2006)

4. Gaston, C., Le Gall, P., Rapin, N., Touil, A.: Symbolic Execution Techniques for Test Purpose Definition. In: Uyar, M.Ü., Duale, A.Y., Fecko, M.A. (eds.) TestCom 2006. LNCS, vol. 3964, pp. 1-18. Springer, Heidelberg (2006)

5. Gotzhein, R., Khendek, F.: Compositional Testing of Communication Systems. In: Uyar, M.Ü., Duale, A.Y., Fecko, M.A. (eds.) TestCom 2006. LNCS, vol. 3964, pp. 227-244. Springer, Heidelberg (2006)

6. Jeannet, B., Jéron, T., Rusu, V., Zinovieva, E.: Symbolic test selection based on approximate analysis. In: Halbwachs, N., Zuck, L.D. (eds.) TACAS 2005. LNCS, vol. 3440, pp. 349-364. Springer, Heidelberg (2005)

7. King, J.-C.: A new approach to program testing. In: Proc. of the Int. Conference on Reliable software, vol. 21(23), pp. 228-233 (1975)

8. Lugato, D., Rapin, N., Gallois, J.-P.: Verification and tests generation for SDL industrial specifications with the AGATHA toolset. In: Larsen, K.G., Nielsen, M. (eds.) CONCUR 2001. LNCS, vol. 2154, pp. 1404-3203. Springer, Heidelberg (2001) ISSN 1404-3203

9. Pelliccione, P., Muccini, H., Bucchiarone, A., Facchini, F.: TeStor: Deriving Test Sequences from Model-based Specification. In: Heineman, G.T., Crnković, I., Schmidt, H.W., Stafford, J.A., Szyperski, C.A., Wallnau, K. (eds.) CBSE 2005. LNCS, vol. 3489, pp. 267-282. Springer, Heidelberg (2005)

10. N. Rapin, C. Gaston, A. Lapitre, and J.-P. Gallois. Behavioural unfolding of formal specifications based on communicating automata. In: Proc. of the 1th Int. Workshop ATVA 2003 (2003) 
11. Tretmans, J.: Conformance Testing with Labelled Transition Systems: Implementation Relations and Test Generation. Computer Networks and ISDN Systems 29, 49-79 (1996)

12. Tretmans, J.: Test generation with inputs, outputs and repetitive quiescence. Software - Concepts and Tools 17(3), 103-120 (1996)

13. van der Bijl, M., Rensink, A., Tretmans, J.: Compositional Testing with IOCO. In: Petrenko, A., Ulrich, A. (eds.) FATES 2003. LNCS, vol. 2931, pp. 86-100. Springer, Heidelberg (2004) 